\title{
Metabolic Pathway and its Relationship to the Biological Activity of some Tuberculostatic and Leprostatic Agents
}

\author{
A. E. WILDER SMITH, B.SC., PII.D., F.R.I.C:, \\ Faculty of Medicine, Genevia
}

It is generally agreed that the metabolic pathway of a drug and its therapeutic activity are related ${ }^{1}{ }^{2}$. Some years $\mathrm{ago}^{3}$ metabolic studics on the two tuberculostatics 4-pyridyl-oxadiazolone ${ }^{5}$ and isoniazid, showed that both substances gave in the rat three identical metabolites. This fact was considered sufficient evidence to draw the conclusion that 4-pyridyloxadiazolone was metabolised in the body via isoniazid, which latter would be responsible for the tuberculostasis. The two drugs would be therefore clinically identical. Cross resistance studics using the two substances against various strains of Mycobacterium tuberculosis showed between 8o-9o per cent cross resistance, confirming this view. Studies on 4-pyridyloxadiazolone $^{5}$ were therefore stopped, it being considered that it was simply another somewhat less active form of isoniazid.

Yet codeine, morphine and heroine are chemically closely related and show similar metabolic pathways in the body. But no one would think of applying them as clinically interchangeable. Their clinical and pharmacological spectra are different and well defined, even though their chemistry and metabolic pathways are so similar. Knowledge of the metabolic pathway is certainly useful, but a great deal of further data such as tissue and organ concentration, chemical stability towards enzymes, partition coefficients, etc., are necessary before attempting to pronounce on the likely clinical spectrum of new substance.

It is proposed here to describe one or two surprises we have experienced in research in the above field in the past few years.

I. If one subcultures $\mathrm{H}_{37} \mathrm{Rv}$ in the presence of just sub-liminal doses of isoniazid, resistance devclops rapidly as shown in Table I. After six subcultures the minimal inhibitory dose of isoniazid has risen from $1: 40$ million to I : 80 thousand.

But if 4-pyridyl-oxadiazolone is cultured under the same conditions against $\mathrm{H}_{37} \mathrm{Rv}$ resistance emergence is much slower (Table I).

2. It seemed, therefore, that the two substances INH and 4-pyridyloxadiazolone were not absolutely biologically identical, at least in vitro, otherwise the resistance emergence rates would have been identical too. If their biological spectra are not absolutely identical, it was thought likcly that, in the presence of 4-pyridyl-oxadiazolone, the resistance emergence rate towards INH would be modified. If the two substances possess exactly the same biological spectrum, a I : I mixture of INH and 4-pyridyl-oxadiazolone should show a resistance emergence rate 
equal to that of INH alone. The pyridyl-oxadiazolone is ten times by weight less active than $\mathrm{INH}$, so that one-tenth of the activity of the mixture will result from it and nine-tenths from isoniazid. 'Table II shows that 4pyridyl-oxadiazolone does modify the resistance emergence rate to INH under in vitro experimental conditions and that therefore the two biological spectra are not totally supcrimposable.

3. The above finding is also applicable to other members of this serics: p-amino-salicylic acid and p-amino-o-hydroxy-phenyl-I, 3, 4-oxadiazolone (WS I 27) show the same activity weight for weight against $\mathrm{H}_{37} \mathrm{Rv}$ and almost complete cross resistance. But when p-amino-salicylic acid and WS $\mathrm{I}_{27}$ in a $\mathrm{I}$ : I mixture are exposed to $\mathrm{H}_{37} \mathrm{Rv}$ subcultures, the resistance emergence rate is lower than that given by p-amino-salicylic acid alone. Sce 'Tables II I and IV.

It would seem, therefore, that, in this scrics of substances at least, cross resistance experiments do not always give exact information on the influence one mycobacteriostatic may have on another.

Onc of the great disappointments of recent years in the chemotherapy of leprosy has been the lack of leprostatic activity shown by isoniazid, in spite of its high tuberculostatic activity. On the basis of metabolic studies alone one would arrive at the conclusion that 4-pyridyl-oxadiazolone would also be inactive towards human leprosy. In fact, however, the oxadiazolone shows quite a high leprostatic activity, and also potentiates the leprostatic activity of Dapsone. ${ }^{4}{ }^{5}$ fact:

There are perhaps three possible explanations of this rather surprising

I. The biological spectrum of pyridyl-oxadiazolone is not ioo per cent superimposable on that of isoniazid, and is leprostatic per se, whereas isoniazid does not possess leprostatic propertics. One cannot settle this hypothesis either way satisfactorily until it has become possible to culture Mycobacterium leprae on a synthetic medium in the presence and in the absence of both substances.

2. Isoniazid possesses chemical properties different to those of 4-pyridyloxadiazolone. The former contains a primary and a tertiary aminogroup, whereas the latter contains two amino and one tertiary aminogroup, and is amphoteric. Isoniazid may therefore be transported in the organism by a different mechanism and route to that traversed by the oxadiazolone. This may mean that isoniazid may not reach the site of Mycobacterium leprae activity, whereas the pyridyl-oxadiazolone may. This fact may have some bearing on the intracellular habit of Mycobacterium leprae. The oxadiazolone is, too, a good deal more stable chemically than isoniazid. For example, it resists hydrolysis much better than the hydrazide. Thus, isoniazid and pyridyl-oxadiazolone may indeed possess similar biological properties, but their chemical and physical properties are widely divergent, which facts may influence their transport at a cellular level.

3. Some leprologists are of the opinion that isoniazid does possess an 
TABle I

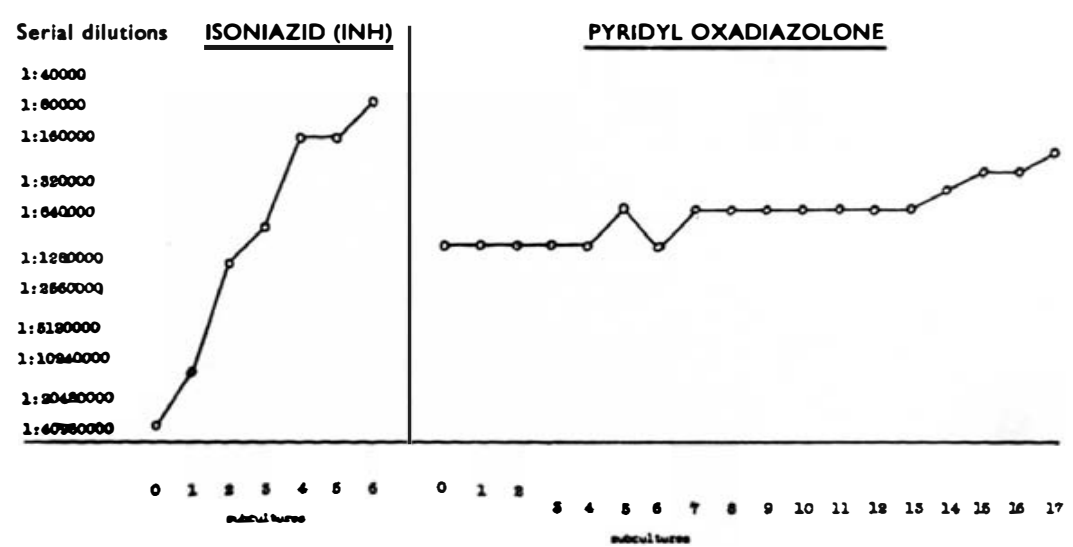

TABLE II

Serial dllutions

2: $\infty 000$

1: $\infty 000$

1: 100000

1: 320000

1: 600000

1:1280 000

2,2060000

2, 8100000

2:10000000

1:0000000

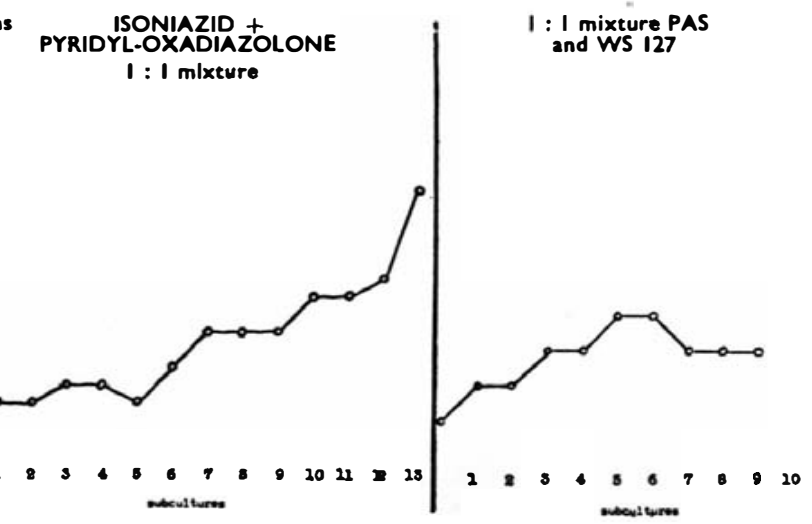

TABle III

: I mixture PAS

I mixture PAs
and WS 127

mentures

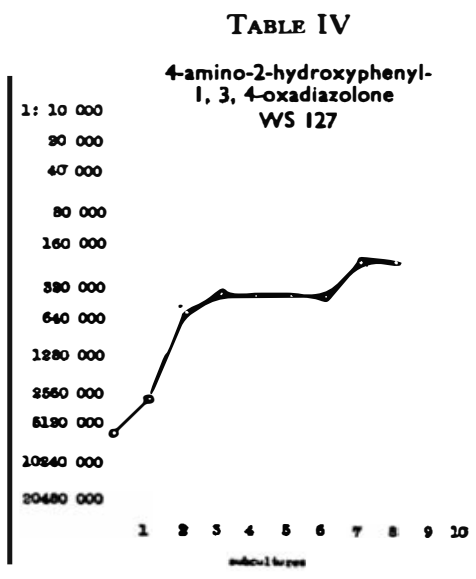


ephemeral action against Mycobacterium leprae, but that after $3^{-10}$ weeks resistance supervenes. The lower rate of resistance emergence against $\mathrm{H}_{37} \mathrm{Rv}$ in the case of pyridyl-oxadiazolone might thus be reflected in its leprostatic activity in the clinic. The purely biochemical explanation of the above may be that the pyridyl-oxadiazolone and its analogues block the enzyme system responsible for the metabolic degradation of isoniazid or other tuberculostatic. In the course of the past three years we have had occasion to extend our observations to other oxadiazolones: p-aminoo-hydroxy-phenyl-I, 3, 4-oxadiazolone (WS 127) shows, as already pointed out, about the same tuberculostatic activity as p-amino-salicylic acid and $80-90$ per cent cross resistance with it. The oxadiazolone is much more slowly eliminated by the human than PAS. Although PAS shows very little leprostatic activity, WS 127 shows, in the few cases of leprosy so far treated with it, a slight but definite leprostatic action. ${ }^{7}$ In tuberculosis WS I27 is a suitable non-toxic, chemically stable and active substitute for PAS, for use in association with isoniazid.

Isoniazid may be converted to 4-pyridyl-I, 3, 4-oxadiazol-thione- 5 (WS 202), by reaction with thiophosgene. ${ }^{8}$ Here again WS 202 is definitely leprostatic, ${ }^{9}$ though less so than its oxygenated analogue.

It would thus seem possible in the few cases so far studied to introduce leprostatic properties to tuberculostatics of the hydrazide class, which are normally devoid of tuberculostatic action, by cyclising the hydrazide group to the corresponding oxadiazolone or oxadiazol-thione. ${ }^{10}$

These relationships are shown in the following schemes:

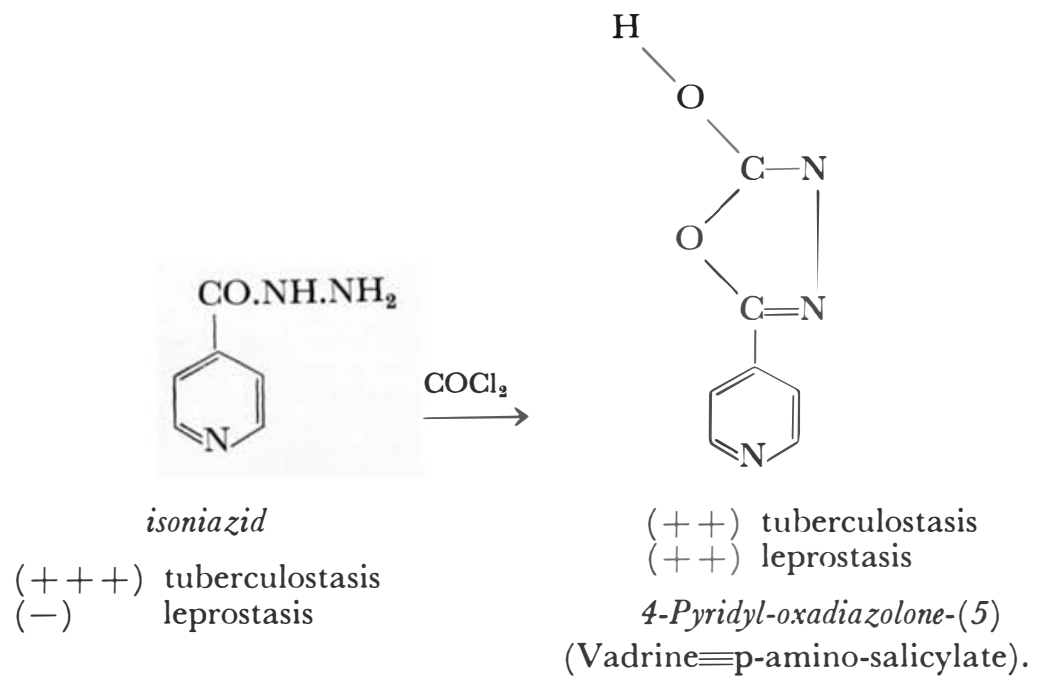




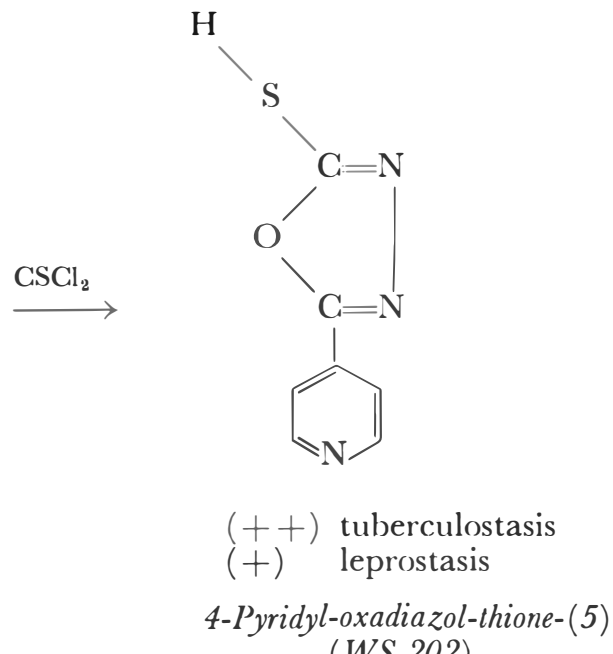

(WS 202)

$\mathrm{H}$<smiles>NNC(=O)c1ccc(N)cc1N</smiles>

p-Amino-salicylic acid hydrazide

p-Amino-o-hydroxy-phenyl-

1,3,4-oxadiazolone-(5)

(WS 127)

$(++)$ tuberculostasis

(-) leprostasis<smiles>Nc1ccc(-c2nnc(Cl)o2)c(O)c1</smiles>

$(++)$ tuberculostasis

$(+)$ leprostasis

Further research is in progress on the above lines.

\section{Literature}

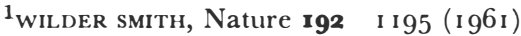

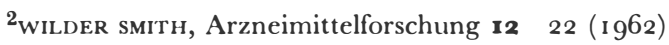

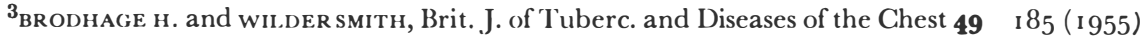

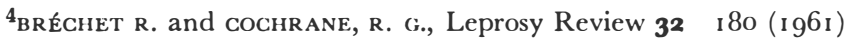


${ }^{5}$ JOPLing W. H. and RIDLeY D. S., Leprosy Review $32 \quad 188$ (196 I)

${ }^{6}$ ShePARD c. c., Leprosy Bacilli in Mouse Foot Pads. Ciba Foundation Study Group No. 15 (1963)

${ }^{7}$ W. H. JOPLING, private communication

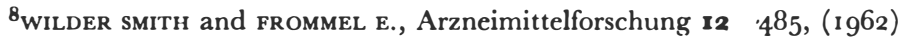

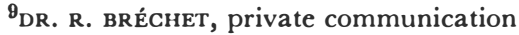

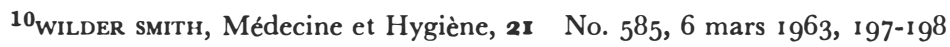

